\title{
Multiple Sclerosis Fatigue is Associated with Reduced Psychomotor Vigilance
}

\author{
D. Rotstein, P. O'Connor, L. Lee, B.J. Murray
}

\begin{abstract}
Background: Fatigue is common and disabling in multiple sclerosis, yet its physiologic substrates remain poorly defined. The aim of this study was to determine the relationship between fatigue and an objective measure of alertness in MS patients. Methods: This study enrolled 49 consecutive MS clinic patients at two academic hospitals in Toronto. Alertness was assessed with the psychomotor vigilance test (PVT), a ten-minute reaction-time test that measures attention and is sensitive to sleep loss. Patients with visual impairment or arm weakness were excluded. Validated tools were used to assess fatigue, disability, mood, and pain. Results: The average age was 43; 65\% were women. Median EDSS was 2.0 (range 0-7.5). Fifty-five percent reported a high impact of fatigue on their lives. Psychomotor vigilance test performance was worse than in an age- and sex-matched population, with a mean reaction time of 315 msecs and 3.98 lapses $>500 \mathrm{msec}(\mathrm{p}<0.001)$. In a multiple regression analysis, fatigue was the most significantly correlated factor with mean PVT reaction time $(\mathrm{p}<0.05)$, and disability was also significantly correlated $(\mathrm{p}<0.01)$. Mood and pain did not correlate with the PVT. Eighteen (37\%) reported often experiencing restlessness in their legs at night. Conclusion: Subjective fatigue and disability were associated with poor performance on alertness testing in MS patients. This research highlights a potential role for psychomotor vigilance testing in providing a standardized assessment tool for an important aspect of MS-related fatigue.
\end{abstract}

RÉSUMÉ: La fatigue dans la sclérose en plaques est associée à une diminution de la vigilance psychomotrice. Contexte : La fatigue est un symptôme fréquent et invalidant chez les patients atteints de la sclérose en plaques (SP) et sa cause physiologique demeure mal définie. Le but de cette étude était de déterminer la relation entre la fatigue et une mesure objective de vigilance chez des patients atteints de la SP. Méthode : Nous avons inclus 49 patients consécutifs de cliniques de SP situées dans deux hôpitaux académiques de Toronto. La vigilance a été évaluée au moyen du test psychomoteur de vigilance (TPV), un test du temps de réaction qui dure dix minutes et qui mesure l'attention, et qui est sensible au manque de sommeil. Les patients qui avaient un déficit visuel ou de la faiblesse au niveau des bras ont été exclus de l'étude. Nous avons utilisé des outils validés pour évaluer la fatigue, l'invalidité, l'humeur et la douleur. Résultats : L'âge moyen des patients était de 43 ans et 65\% étaient des femmes. Le score médian à l'EDSS était de 2,0 (écart de 0 à 7,5). Cinquante-cinq pour cent ont rapporté que la fatigue avait des conséquences importantes sur leur vie. Leur performance au test psychomoteur de vigilance était moins bonne que celle d'une population de sujets appariés pour l'âge et le sexe, le temps moyen de réaction étant de $315 \mathrm{msec}$ dont 3,98 > $500 \mathrm{msec}(\mathrm{p}<0,001)$. À l'analyse de régression multiple, la fatigue était le facteur dont la corrélation avec le temps de réaction au TPV était le plus significatif $(\mathrm{p}<0,05)$ et il en était de même pour l'invalidité $(\mathrm{p}<0,01)$. L'humeur et la douleur n'étaient pas corrélés au TPV. Dixhuit $(37 \%)$ des patients ont rapporté qu'ils avaient des impatiences dans les jambes la nuit. Conclusion : La fatigue subjective et l'invalidité étaient associées à de moins bons résultats au test de vigilance chez les patients atteints de la SP. Cette étude souligne le rôle que pourrait jouer le test de psychomoteur de vigilance comme outil d'évaluation standard d'un aspect important de la fatigue reliée à la SP.

Can J Neurol Sci. 2012; 39: 180-184

Fatigue is a common and disabling symptom in those who suffer from multiple sclerosis (MS). Studies indicate that more than $75 \%$ of MS patients suffer from fatigue. ${ }^{1-3}$ Two-thirds of MS patients rank fatigue as one of their three most debilitating symptoms. ${ }^{1}$ Multiple sclerosis fatigue is particularly aggravated by heat and can significantly impair physical activity. ${ }^{1}$ It is one of the main causes of work absenteeism in individuals with MS. ${ }^{4}$ Yet fatigue is a lay term that remains subjectively defined and assessment of fatigue is therefore dependent on self-report. Perhaps as a result, the physiologic substrates of MS fatigue are not well-described. The subjective experience of fatigue is multifactorial and has been correlated with factors such as disability, depression, and pain. ${ }^{5-7}$ Interestingly, no clear association has been established between MS fatigue and demographic factors, such as age and gender; lesion burden; or the clinical form of MS. ${ }^{1,7-9}$
One of the first studies that looked at fatigue in MS found that $43 \%$ of subjects identified "sleepiness" as part of the symptomatology of their fatigue. ${ }^{2}$ It is known that some sleep disorders, in particular restless legs syndrome, are more prevalent in the MS population. ${ }^{10-13} \mathrm{~A}$ pilot study involving polysomnography of $12 \mathrm{MS}$ patients suggested differences in sleep quality in patients with MS compared to controls, and a

\footnotetext{
From the Division of Neurology, Department of Medicine (DR, PO, LL, BJM), University of Toronto; St. Michael's Hospital (PO); Sunnybrook Health Sciences Centre (LL, BJM), Toronto, Ontario, Canada.

Received March 23, 2011. Final Revisions Submitted October 11, 2011 Correspondence to: Brian Murray, M1600, Sunnybrook Health Sciences Centre, 2075 Bayview Avenue, Toronto, Ontario, M4N 3M5, Canada.
} 
trend for increased sleepiness as measured objectively with the multiple sleep latency test. ${ }^{14}$ Several small studies have found that treatment with modafinil, a wake-promoting agent, improved symptoms of MS fatigue, although in a larger randomized controlled trial there was no difference. ${ }^{15-17}$ These studies did not use objective measures of alertness. Beyond these pharmaceutical studies, little research has been conducted into whether poor sleep and decreased alertness are major determinants of MS fatigue. It is important to clarify the contribution of sleep deprivation and poor alertness to MS fatigue as they may be more easily treated than other factors, such as disability.

In this study we set out to determine the relationship in MS between subjectively reported fatigue and objective sleepiness as evaluated by the psychomotor vigilance test (PVT). The PVT is a reaction-time task that has been validated in laboratory, clinical, and field studies investigating sleep, respiratory, and neurological disorders. ${ }^{18-20}$ The PVT has been demonstrated to be highly sensitive to sleep loss and can easily be used in a clinical setting. We also assessed other factors, including demographics, disability, pain, mood, and sleep quality, to assess their relative contribution to psychomotor vigilance performance in this population.

\section{Methods}

This was a prospective cohort study that enrolled 49 consecutive patients presenting to the MS clinics at two major Toronto teaching hospitals (St. Michael's Hospital and Sunnybrook Health Sciences Centre). Approval was obtained from the research ethics board at each hospital in advance of the study. Written informed consent was obtained from all participants. Exclusion criteria were self-report of visual impairment or any degree of hand or arm weakness.

Alertness was assessed using the PVT, which is a noninvasive reaction time task of ten minutes duration. It requires the subject to hold a small apparatus and press a button when a light signal appears on the screen of the device. The PVT measures reaction times over variable intervals of seconds between visual stimuli. Subjects are allowed a brief trial with the apparatus before the test begins to ensure that they understand the instructions. Key variables collected by the device include mean reaction time and number of lapses (response delays $>500$ $\mathrm{msec}$ ). There is minimal learning effect on performance, as shown in other studies. ${ }^{21}$

The primary outcome of interest was the mean reaction time (in milliseconds) on the PVT. Mean reaction times in the general population have been previously published, by gender and by age group. ${ }^{19}$ The mean reaction time for a general population sample with the same gender and age distribution as the study population was estimated from these data. Self-reporting of fatigue was assessed using the Modified Fatigue Impact Scale (MFIS), an instrument that consists of 21 questions measuring social, physical, and cognitive aspects of fatigue. ${ }^{22}$ Physical disability was assessed with the Expanded Disability Status Scale (EDSS) based on the patients' neurological examination findings, as documented by their own neurologist, on the day of the visit. Mood was graded with the Beck Depression Inventory fast screen (BDI-FS) for medically ill patients, and pain with the Medical Outcomes Study (MOS) Pain Effects Scale (PES); both are self-report questionnaires. The MFIS, EDSS, BDI-FS, and MOS PES are all validated instruments that are wellcharacterized in the MS population. ${ }^{22-25}$ Subjective sleepiness was evaluated using the Epworth Sleepiness Scale (ESS), which is a validated widely-used measure in the sleep literature. ${ }^{26}$ Subjective sleep characteristics were assessed using a sleep questionnaire for common sleep habits. Variables of interest included duration of sleep, as well as restless legs, sleepdisordered breathing, and narcolepsy symptoms.

Restless legs was defined as often or always having a selfreported feeling of discomfort in the legs at night that improved with movement. Sleep-disordered breathing was suspected if the subject reported that $\mathrm{s} / \mathrm{he}$ often or always snored at night. Narcolepsy symptoms were defined by self-reporting of sleepiness and at least two out of the following three symptoms: 1) muscular weakness associated with strong emotions; 2) sleep paralysis; and 3) hypnagogic or hypnapompic hallucinations.

Statistical analysis was carried out using the SPSS-16 program. We performed a stepwise multiple regression analysis to determine the best predictors of objective alertness, as measured by the PVT. Factors considered were gender, EDSS, BDI-FS, MFIS, PES, restlessness, duration of sleep, and ESS.

\section{Results}

The average age of subjects was $43(+11)$, and $65 \%$ were women (Table 1). The median EDSS was 2.0 (range 0-7.5). Twenty-seven $(55 \%)$ reported a high impact of fatigue on their lives. Five $(10 \%)$ were on pharmaceutical treatment for fatigue with amantadine or modafinil. Twenty-eight (57\%) were on disease modifying therapy and 13 (26.5\%) were on an antidepressant.

The mean reaction time on the PVT was $315.0(+65.1)$ with $90 \%$ of subjects demonstrating reaction times exceeding the

Table 1: Sample demographics and descriptive data $(n=49)$

\begin{tabular}{|c|c|}
\hline Characteristic & Mean + SD \\
\hline Age (years) & $42.7 \pm 10.7$ \\
\hline Men, $\%$ & 34.7 \\
\hline Married, $\%$ & $55.1 \%$ \\
\hline Education: 12 years or more & $97.9 \%$ \\
\hline Income: $>\$ 60,000$ & $60.6 \%$ \\
\hline Weekly Hours of Paid Employment & $24.7+20.2$ \\
\hline Years Since Diagnosis & $8.7 \pm 8.5$ \\
\hline On modafinil or amantadine & $10.2 \%$ \\
\hline On disease modifying therapy & $57.1 \%$ \\
\hline On an antidepressant & $26.5 \%$ \\
\hline Expanded Disability Status Scale (EDSS) & median 3.5 (range 0-7.5) \\
\hline Beck Depression Inventory (BDI-FS) $^{1}$ & median 3 (range $0-11$ ) \\
\hline Modified Fatigue Impact Scale (MFIS) $^{2}$ & median 41 (range 1-75) \\
\hline Pain Effects Scale (PES) & median 13 (range 6-28) \\
\hline Epworth Sleepiness Scale (ESS) $^{3}$ & median 6 (range $0-18)$ \\
\hline PVT mean reaction time $(\mathrm{ms})$ & $315.0 \pm 65.1$ \\
\hline PVT lapses & $3.98 \pm 5.24$ \\
\hline
\end{tabular}

1 Depression suggested where BDI-FS $>4$; 2 High impact of fatigue where MFIS $>39 ; 3$ Pathologic sleepiness where ESS $>10$ 


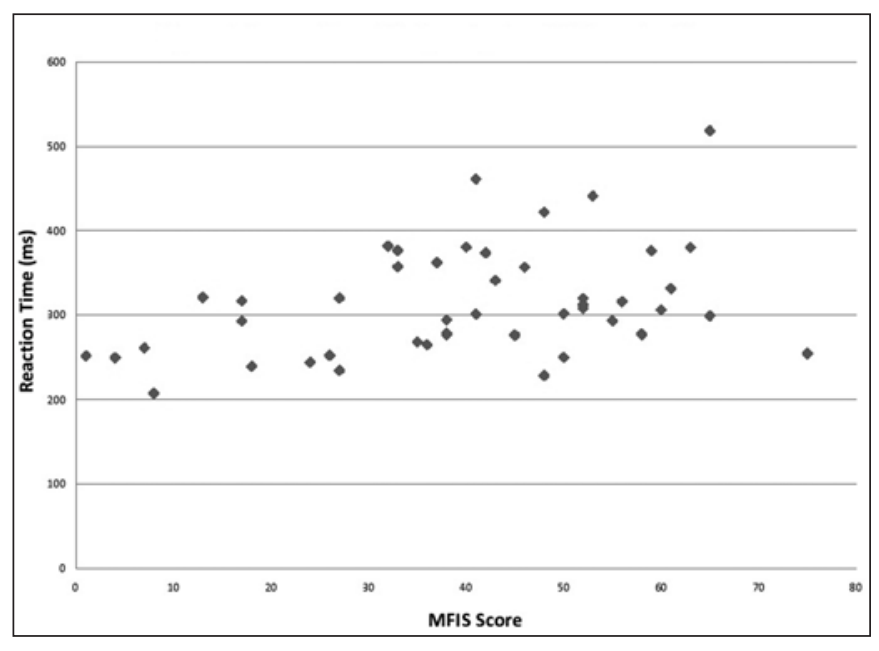

Figure: The figure depicts MS patients' self-reported fatigue scores, as assessed via the MFIS, plotted against mean reaction time on the PVT. Abbreviations: MFIS = Modified Fatigue Impact Scale.

mean $(246 \mathrm{msec})$ of an age- and sex-matched control population $(\mathrm{p}<0.0001) .{ }^{19}$ The mean number of lapses was $3.98(+5.24)$ with $59 \%$ exceeding the mean (1.96) for an age- and sex-matched control $(\mathrm{p}=0.001) .^{19}$

The Figure, a scatter plot of MFIS score against PVT reaction time, demonstrates the correlation between reaction times and fatigue scores. The Pearson correlation coefficient was 0.38 . The correlation coefficient (R) for the MFIS observed in our multiple regression model was similar at $0.365(\mathrm{p}<0.05)$ (Table 2$)$.

Thirty percent endorsed sleepiness on the subjective ESS exceeding the normal threshold (10). ${ }^{26}$ Eighteen (37\%) described often or always having restlessness in their legs at night that improved with movement. Six (12\%) endorsed snoring, suggesting sleep-disordered breathing. Seven (14\%) subjects reported narcolepsy symptoms. These three groups of sleepdisordered patients each displayed prolonged average reaction times and higher MFIS scores compared to the rest of the MS patients, although these trends did not meet statistical significance.

In a stepwise regression model, fatigue (MFIS) showed the strongest correlation with PVT reaction time $(\mathrm{p}<0.05)$ (Table 2). Other predictors of objective alertness were EDSS $(p<0.01)$, duration of sleep $(\mathrm{p}=0.01)$, and female gender $(\mathrm{p}=0.01)$. Mood, pain, subjective sleepiness (high ESS scores), and restless legs were not associated with impaired objective alertness in this model.

\section{Discussion}

This study demonstrated objective impairments in alertness using the psychomotor vigilance test, and a moderate correlation between alertness and subjectively reported fatigue in MS patients. The PVT has previously been shown to be sensitive to the benefits of bright light, naps, and caffeine in sleepy individuals and may be used to track treatment response. The PVT is a rapid test with little training required for either subject or administrator. Results do not vary with educational background.$^{21}$ It is associated with a minimal learning curve and has high test-retest reliability. ${ }^{21}$ It offers an advantage over previously validated fatigue assessment tools in that it does not rely on self-report.

Furthermore, the PVT may offer unique benefits particularly for evaluating the impact of interventions on fatigue in an immediate way. The MFIS asks respondents to rate how much fatigue has contributed to each of a set of listed statements over the preceding four weeks. Other fatigue rating scales similarly elicit information about the impact of fatigue on activities over long periods of time. These rating scales need to designate a

Table 2: Summary of stepwise regression model

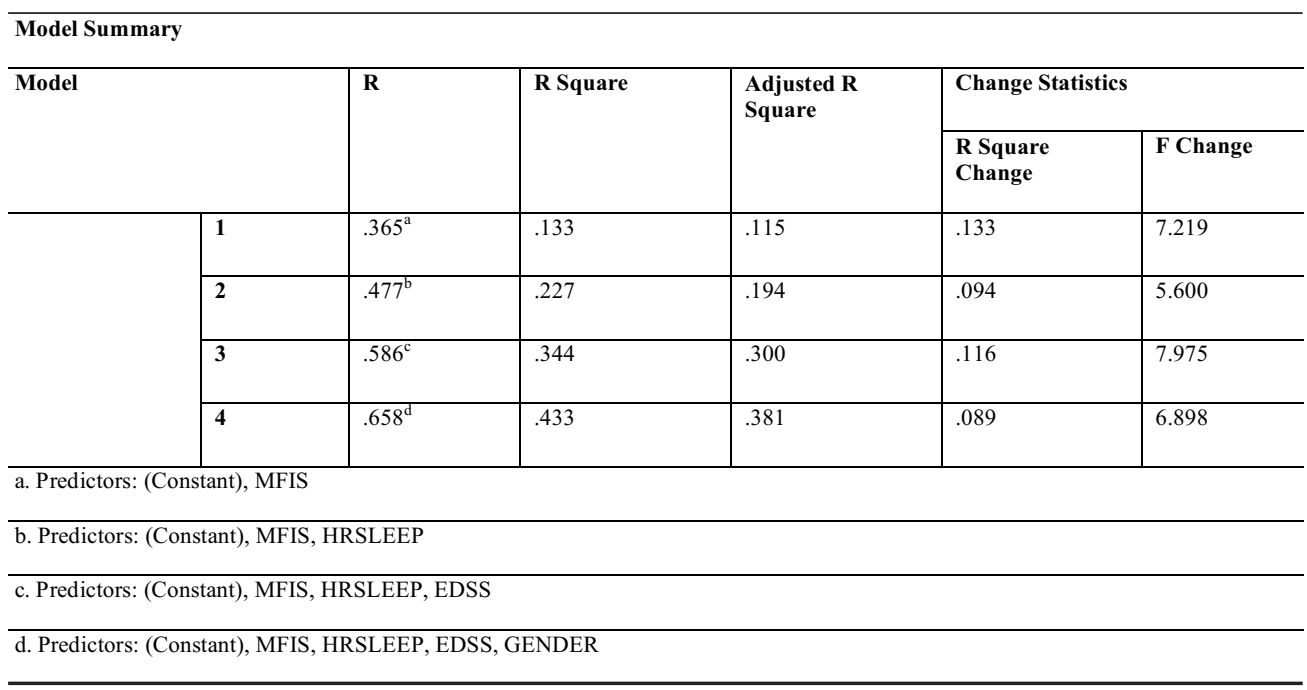


sufficiently long period that the subject's reported fatigue is not swayed by a few days of relative under- or over-exertion. However, their imposition of a longer timeframe may bias results as other extraneous factors become more important than the intervention in determining the subject's fatigue. For example, it may be difficult to prove that recent exertion, changes in temperature, or recently administered medications impact the MFIS, but the PVT allows for an immediate and focused outcome measure.

In this study, fatigue scores, level of disability, longer sleeping times, and female gender were all associated with poor reaction time performance in MS patients; mood, pain, restless legs, and self-reported sleepiness were not. Mood has been characterized as a contributor to MS fatigue in many studies. ${ }^{5-7}$ However, our findings suggest that mood may affect the perception of fatigue more than its cognitive correlates in MS. This hypothesis is supported by a recent study showing that MS patients' self-rankings on the Beck Depression Inventory Fast Screen were moderately correlated with rankings on the Fatigue Severity Scale, but not with cognitive results from formal neuropsychological testing. ${ }^{27}$ Pain may also be more influential on perceptions of fatigue than on performance measures. However, the PVT is a simple task and it is possible that with a more complex, multi-step task these factors would be associated with a decline in performance. This could be an interesting direction for future research.

The finding of impaired alertness with more sleep was paradoxical. In a general population longer sleeping times correlate with better alertness. ${ }^{21}$ The opposite finding here may reflect an underlying association between somnolence and a high lesion load, or somnolence resulting from poor quality sleep in MS. Assessing lesion burden was not within the scope of this study, but should be pursued in future investigations into MSrelated fatigue.

We found that restless leg and narcolepsy symptoms were prevalent in this MS population. The reason for this propensity may be related to lesion location, HLA-type, or other immunological mechanisms. ${ }^{12,21,28}$ Sleep-disordered breathing tended to be associated with increased fatigue and poor alertness as it would in the general population. This finding did not meet statistical significance likely because of the relative rarity $(n=6)$ of self-reported sleep-disordered breathing symptoms in this population. Restless legs symptoms $(n=18)$ were common in this population, as has been reported for other groups of subjects with MS. ${ }^{10-12}$ It is difficult to determine whether the lack of difference in reaction times between the subjects with restless leg symptoms and the other MS subjects was related to the subjective reporting of symptoms or to a true sparing of psychomotor vigilance. It would be worthwhile to explore psychomotor vigilance in MS subjects for whom sleep disturbances were objectively quantified with polysomnography.

\section{Conclusion}

The PVT reaction time offers considerable potential as an objective parameter for evaluating fatigue in MS patients. Reaction time performance was moderately correlated with the MFIS, a validated instrument for measuring fatigue in the MS population. The concept of fatigue, however, clearly includes more than impaired alertness. The PVT does not entirely capture features of depressed mood or pain, for example. Mood has been demonstrated in previous research to be correlated with MS fatigue, but did not significantly affect reaction times in this study. ${ }^{4-5,7}$ The PVT can be influenced by demographic factors that have not been consistently linked to MS-related fatigue. For example, in other studies age has been shown to affect PVT performance, and in this study, gender was a significant contributor. ${ }^{19}$ However, these observations also illustrate the drawback of traditional fatigue rating scales, which encompass several distinct parameters - short-term attention, exercise tolerance, sustained concentration capabilities - under the umbrella of fatigue. Perhaps it will be fruitful in the future to research MS fatigue by differentiating this entity into several, smaller, objectively defined components, including alertness. The PVT thus may prove very useful as a quantitative assessment tool of this important aspect of MS fatigue and help to identify and evaluate novel treatment targets.

\section{ACKNOWLEDGEMENTS}

This study was performed at St. Michael's Hospital and the Sunnybrook Health Sciences Centre in Toronto, Canada.

\section{Disclosures}

Dr. Dalia Rotstein completed the data analysis.

Dr. Liesly Lee has received either personal compensation (for consulting, serving on a scientific advisory board, or speaking) or financial support for scholarly activities from pharmaceutical companies that develop products for MS, including Biogen Idec., Sanofi-Aventis, EMD Serono, Teva Pharmaceuticals, Bayer, Bio MS, Roche, and Novartis.

Dr. Paul O'Connor has received either personal compensation (for consulting, serving on a scientific advisory board, or speaking) or financial support for scholarly activities from pharmaceutical companies that develop products for MS, including Biogen Idec., Sanofi-Aventis, EMD Serono, Abbott Labs, Teva Pharmaceuticals, Bayer, Bio MS, Genentech, Roche, and Novartis.

Dr. Brian Murray has received personal compensation (for consulting, serving on a scientific advisory board, or speaking) from Pfizer, Valeant, and UCB Pharma. 


\section{REFERENCES}

1. Krupp LB. Fatigue in multiple sclerosis: a guide to diagnosis and management. New York: Demos Medical Publishing Inc.; 2004.

2. Freal JE, Kraft GH, Coryell JK. Symptomatic fatigue in multiple sclerosis. Arch Phys Med Rehabil. 1984;65:135-8.

3. Fisk JD, Pontefract A, Ritvo PG, et al. The impact of fatigue on patients with multiple sclerosis. Can J Neurol Sci. 1994;21(1):914.

4. Ziemssen T. Multiple sclerosis beyond EDSS: depression and fatigue. J Neurol Sci. 2009;277 S1:S37-41.

5. Bakshi R, Shaikh ZA, Miletich RS, et al. Fatigue in multiple sclerosis and its relationship to depression and neurological disability. Mult Scler. 2000;6:181-5.

6. Patrick E, Christodoulou C, Krupp LB. Longitudinal correlates of fatigue in multiple sclerosis. Mult Scler. 2009;15(2):258-61.

7. Kroenke DC, Lynch SG, Denney DR. Fatigue in multiple sclerosis: relationship to depression, disability, and disease pattern. Mult Scler. 2000;6:131-6.

8. Bakshi R, Miletich RS, Henschel K, et al. Fatigue in multiple sclerosis: cross-sectional correlation with brain MRI findings in 71 patients. Neurology. 1999;53:1151-3.

9. Iriarte J, Katsamakis G, de Castro P. The Fatigue Descriptive Scale (FDS): a useful tool to evaluate fatigue in multiple sclerosis. Mult Scler. 1999;5:10-6.

10. Deriu M, Cossu G, Molari A, et al. Restless legs syndrome in multiple sclerosis: a case-control study. Mov Disord. 2009;24 (5):697-701 .

11. Manconi M, Fabbrini M, Bonanni E, et al. High prevalence of restless legs syndrome in multiple sclerosis. Eur J Neurol. 2007; 14(5):534-9.

12. Manconi M, Ferini-Strambi L, Filippi M, et al. Multicenter case control study on restless legs syndrome. Sleep. 2008;31(7): 944-52.

13. Brass SD, Duquette P, Proulx-Therrien J, Auerbach S. Sleep disorders in patients with multiple sclerosis. Sleep Med Rev. 2010;14(2):121-9.

14. Beran RG, Ainley LAE, Holland G. Sleepiness in multiple sclerosis: A pilot study. Sleep Biol Rhythms. 2008;6:194-200.

15. Zifko UA, Rupp M, Schwarz S, Zipko HT, Maida EM. Modafinil in treatment of fatigue in multiple sclerosis. Results of an openlabel study. J Neurol. 2002;249(8):983-7.
16. Stankoff B, Waubant E, Confavreaux C. Modafinil for fatigue in MS: a randomized, placebo-controlled, double-blind trial. Neurology. 2005;64(7):1139-43.

17. Brioschi A, Gramigna S, Werth E, et al. Effect of modafinil on subjective fatigue in multiple sclerosis. Eur Neurol. 2009;62(4): 243-9.

18. Lim J, Dinges DF. Sleep deprivation and vigilant attention. Ann N Y Acad Sci. 2008;1129:305-22.

19. Kim H, Dinges DF, Young T. Sleep-disordered breathing and psychomotor vigilance in a community-based sample. Sleep. 2007;30(10):1309-16.

20. Adam M, Retey JV, Khatami R, Landolt HP. Age-related changes in the time course of vigilant attention during 40 hours without sleep in men. Sleep. 2006;29(1):55-7.

21. Dorrian J, Rogers NL, Dinges DF. Psychomotor vigilance performance: a neurocognitive assay sensitive to sleep loss. In Kushida CA, ed. Sleep Deprivation. New York: Marcel Dekker, Inc.; 2005.

22. Multiple sclerosis clinical practice guidelines: fatigue and multiple sclerosis evidence-based management strategies for fatigue in multiple sclerosis. Washington, DC: Paralyzed Veterans Association; 1998.

23. Kurtzke JF. Rating neurologic impairment in multiple sclerosis: an expanded disability status scale (EDSS). Neurology. 1983;33: 1444.

24. Benedict RH, Fishman I, McClellan MM, Bakshi R, WeinstockGuttman B. Validity of the Beck Depression Inventory-fast screen in multiple sclerosis. Mult Scler. 2003;9(4):393-6.

25. Fischer JS, Rudick RA, Cutter GR, Reingold SC. The Multiple Sclerosis Functional Composite Measure (MSFC): an integrated approach to MS clinical outcome assessment. Mult Scler. 1999; 5(4):244-50.

26. Johns MW. Reliability and factor analysis of the Epworth Sleepiness Scale. Sleep. 1992;15:376-81.

27. Morrow SA, Weinstock-Guttman B, Munschauer FE, Hojnacki D, Benedict RH. Subjective fatigue is not associated with cognitive impairment in multiple sclerosis: cross-sectional and longitudinal analysis. Mult Scler. 2009;15(8):998-1005.

28. Kanbayashi T, Shimohata T, Nakashima I, et al. Symptomatic narcolepsy in patients with neuromyelitis optica and multiple sclerosis: new neurochemical and immunological implications. Arch Neurol. 2009;66(12):1563-6. 